\title{
Establishing the State Function to Determine the Turning Radius of the Vehicle When Steering
}

\author{
Nguyen Tuan Anh ${ }^{1, *}$, Hoang Thang Binh ${ }^{2}$, Tran Van Thang ${ }^{3}$ \\ ${ }^{1}$ Department of Mechatronics-Automobile, Hanoi University of Business and Technology, Vietnam \\ ${ }^{2}$ School of Transportation Engineering, Hanoi University of Science and Technology, Vietnam \\ ${ }^{3}$ Department of Mechanical Engineering, Hanoi Vocational College of Technology, Vietnam
}

Received February 24, 2020; Revised March 16, 2020; Accepted March 28, 2020

Copyright $\mathrm{C} 2020$ by authors, all rights reserved. Authors agree that this article remains permanently open access under the terms of the Creative Commons Attribution License 4.0 International License

\begin{abstract}
The turning radius of the vehicle is determined based on the coordinates of the center of gravity. When the vehicle is moving at different velocities and driving at different steering angles, the value of the turning radius is also different. This change is nonlinear and continuous and determined by simulation or experimentation process. Previous studies have not established the formula to calculate the value of a turning radius of the vehicle based on input parameters. Therefore, determining the value of a turning radius is complex and time-consuming. This research has shown the dependence between turning radius $\mathrm{R}$ on input parameters such as velocity $\mathrm{v}$ and steering angle $\delta$ by using a double-track dynamic vehicle model to simulate. Besides, this research also established the state function to calculate the turning radius of the vehicle at different velocities and steering angles. The state function is determined based on the rule of change of the corresponding values. Using only the determined input parameters like velocity and steering angle, the value of the turning radius can be easily calculated with high accuracy through 4 steps. The reference coefficients needed to calculate are also provided in this research. The results of the research can be applied in various computational conditions.
\end{abstract}

Keywords State Function, Turning Radius, Steering, Dynamic Vehicle

\section{Introduction}

\subsection{The Problem of the Vehicle Stability}

Automobiles are popular vehicles used around the world. Today, with the development of technology and engineering, automobiles have also been greatly improved. Besides, the quality of roads and facilities is also much more modern than before. In fact, the velocity of vehicles is very high. Therefore, the problems of stability and safety are always the top priority.

The problems of safety and stability of the vehicle are considered when the vehicle accelerates, brakes or steers. In particular, the case of steering is always rated as the most dangerous. When the vehicle is moving at high velocity and sudden steering, the vehicle may encounter several cases such as sideslip, rollover, or deviating trajectory [1]. Therefore, this problem greatly affects the safety of passengers. This research focuses on the problems of determining the vehicle's trajectory when steering. This parameter is shown by the value of the turning radius $R$.

\subsection{Literature Review}

Previous studies have often focused on determining the turning radius in a static state [2-6]. This is the value of the minimum turning radius, it is determined based on the size of the vehicle and the maximum steering angle as formula below.

$$
\begin{gathered}
R=\frac{a_{1}+a_{2}}{\tan \delta_{\text {max }}} \\
\cot \delta_{1}-\cot \delta_{2}=\frac{2 b}{a_{1}+a_{2}}
\end{gathered}
$$

Also, many studies on improving steering effectiveness have been conducted and introduced [7-10]. To determine the turning radius of the vehicle, various dynamic models were used as single-track, double-track,... [11-14].

However, most studies have only determined a minimum turning radius in a stationary state. In fact, the value of the turning radius in the dynamic state makes more sense than in the static state. To determine this value, the dynamic models of the vehicle need to be used to simulate the movements of the vehicle, which is time-consuming 
and inconvenient. Therefore, this research proceeds to establish the state function to easily calculate the turning radius of the vehicle in a dynamic state based on the defined input parameters at different conditions.

\section{Methodology}

\subsection{The Double-track Dynamic Model}

To determine the vehicle's trajectory, the double-track dynamic model was used (Figure 1).

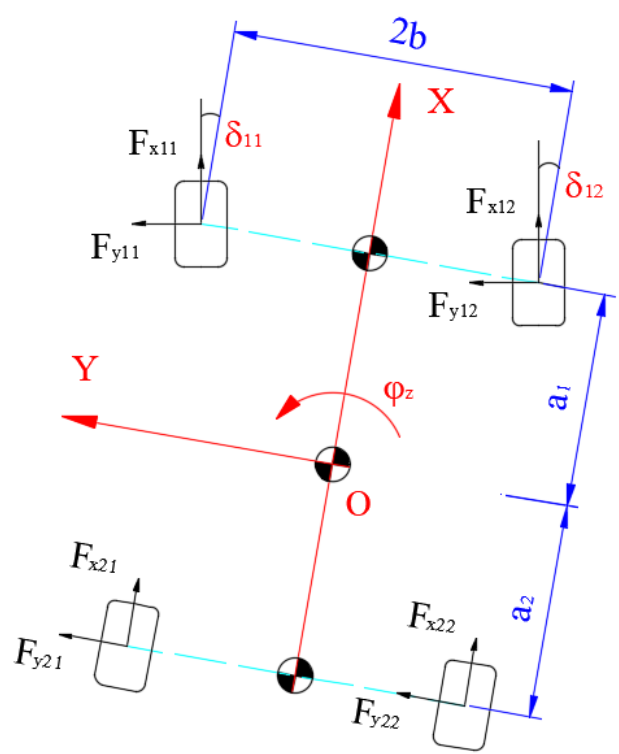

Figure 1. The double-track dynamic model

The equations describing the motion of the vehicle are given below:

$$
\begin{gathered}
M\left(\dot{v}_{x}-\dot{\varphi}_{z} v_{y}\right)=F_{x 11} \cos \delta_{11}+F_{x 12} \cos \delta_{12} \\
-F_{y 11} \sin \delta_{11}-F_{y 12} \sin \delta_{12}+F_{x 21}+F_{x 22} \\
M\left(\dot{v}_{y}+\dot{\varphi}_{z} v_{x}\right)=F_{x 11} \sin \delta_{11}+F_{x 12} \sin \delta_{12} \\
+F_{y 11} \cos \delta_{11}+F_{y 12} \cos \delta_{12}+F_{y 21}+F_{y 22} \\
I_{z} \ddot{\varphi}_{z}=\left(\begin{array}{l}
-F_{x 11} \cos \delta_{11}+F_{x 12} \cos \delta_{12}+F_{y 11} \sin \delta_{11} \\
-F_{y 12} \sin \delta_{12}-F_{x 21}+F_{x 22}
\end{array}\right) b+ \\
\left(\begin{array}{l}
F_{x 11} \sin \delta_{11}+F_{y 11} \cos \delta_{11}+ \\
F_{x 12} \sin \delta_{12}+F_{y 12} \cos \delta_{12}
\end{array}\right) a_{1}-\left(F_{y 21}+F_{y 22}\right) a_{2}
\end{gathered}
$$

Where:

$M$ : Mass of the vehicle.

$I_{z}$ : Moment of inertia of the $\mathrm{z}$-axis.

$\varphi_{z}$ : Yaw angle.

$F_{x i j}$ : Longitudinal force of the wheels.

$F_{y i j}$ : Lateral force of the wheels. $\delta_{i j}$ : Steering angles.

$a_{1}$ : Distance from center of gravity to font axle.

$a_{2}$ : Distance from center of gravity to rear axle.

$2 b$ : Base width.

$v_{x}$ : Longitudinal velocity of the vehicle.

$v_{y}$ : Lateral velocity of the vehicle.

The values of the longitudinal forces $F_{x i j}$ and lateral forces $F_{y i j}$ of the wheels are determined by the Pacejka tire model [15].

The trajectory of the vehicle is determined by its center coordinates:

$$
\begin{aligned}
x & =\int_{0}^{t} v \cos \left(\beta+\varphi_{z}\right) d t \\
y & =\int_{0}^{t} v \sin \left(\beta+\varphi_{z}\right) d t
\end{aligned}
$$

The heading angle of the vehicle $\beta$ is calculated based on the formula below:

$$
\beta=\arctan \frac{v_{y}}{v_{x}}
$$

\subsection{Conditions of the Simulation Process}

Simulation is carried out under the following conditions:

- Vehicle moves at a stable velocity, the value of the velocity is about $5-90(\mathrm{~km} / \mathrm{h})$.

- Vehicle steers with a small steering angle $\left(\delta \leq 5^{0}\right)$, assume that $\delta=\delta_{11}=\delta_{12}$. When $\delta$ changes, $\omega=$ const ( $\omega$ : steering velocity).

- Specification of the vehicle is given as in table 1 .

The simulation process is performed by Matlab-Simulink software based on the selected parameters and conditions.

Table 1. Specification of the vehicle.

\begin{tabular}{|c|c|c|}
\hline Symbol & Value & Unit \\
\hline$M$ & 1880 & $\mathrm{~kg}$ \\
\hline$I_{z}$ & 2200 & $\mathrm{kgm}^{2}$ \\
\hline$b$ & 750 & $\mathrm{~mm}$ \\
\hline$a_{1}$ & 1110 & $\mathrm{~mm}$ \\
\hline$a_{2}$ & 1650 & $\mathrm{~mm}$ \\
\hline
\end{tabular}

\section{Establishing the State Function and Results}

\subsection{Results of the Simulation Process}

Using the parameters given in Table 1, the turning radius of the vehicle when at different velocities and steering angles is described in Figure 2. 


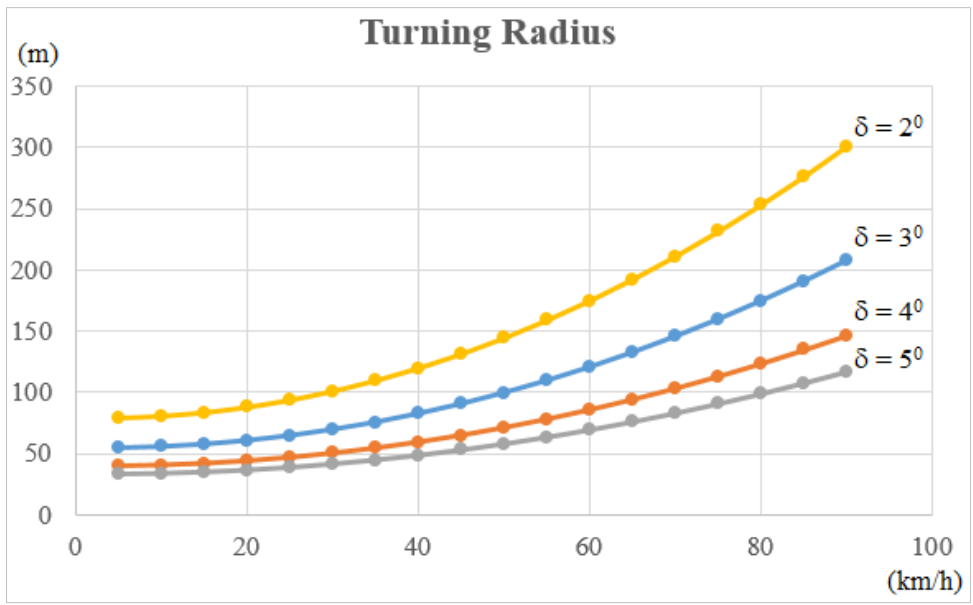

Figure 2. Dependence of turning radius $R$ on velocity $v$

The graph in Figure 2 indicates that:

When the velocity increases (steering angle is constant), the turning radius will increase. The graph of the value of the turning radius is a nonlinear curved line. If the steering angle is small, the change of the turning radius will be large.

When the steering angle increases (velocity is constant), the turning radius decreases.

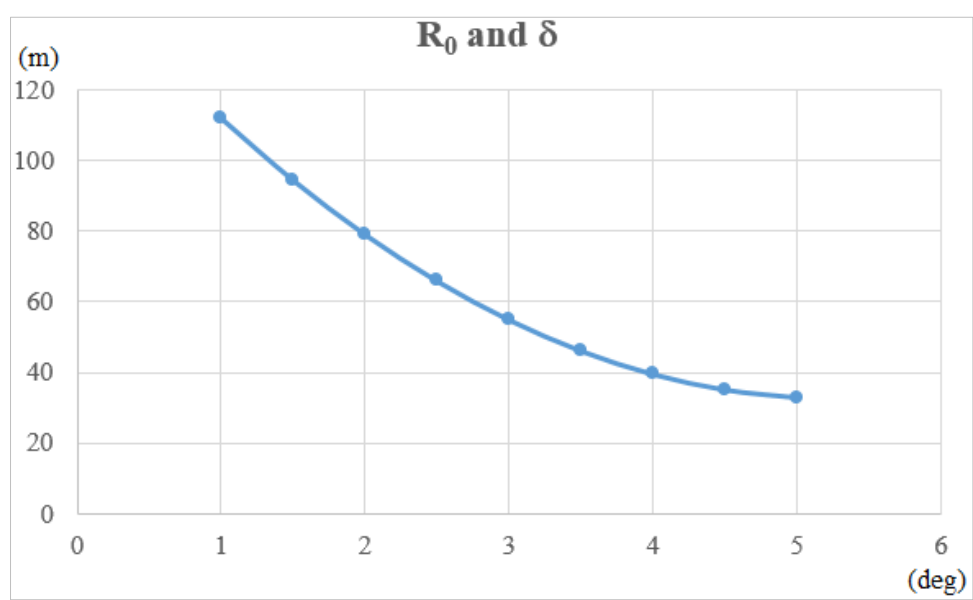

Figure 3. Dependence of initial turning radius $R_{0}$ on steering angle $\delta$

The graph in Figure 3 shows the dependence between the initial turning radius $R_{0}$ and the steering angle $\delta$. If the steering angle $\delta$ increases, the initial turning radius $R_{0}$ will decrease. This graph is a nonlinear curved line with a decreasing slope as the value of the steering angle $\delta$ increases.

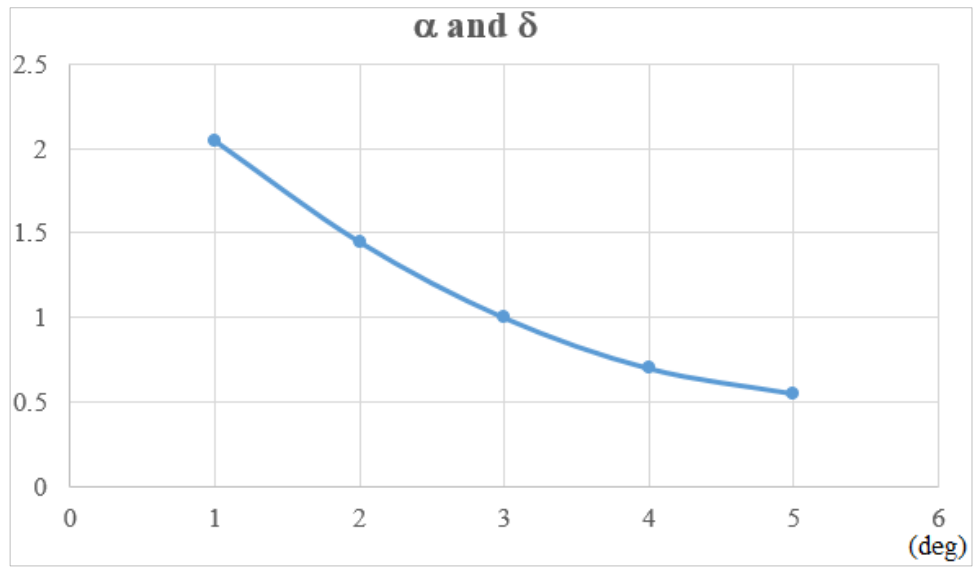

Figure 4. Dependence of coefficient $\alpha$ on steering angle $\delta$ 
The graph in Figure 4 shows the dependence between the coefficient $\alpha$ and the steering angle $\delta$. If the steering angle $\delta$ increases, the value of the coefficient $\alpha$ will decrease. This graph is a nonlinear curve with a decreasing slope as the value of the steering angle $\delta$ increases. The coefficient $\alpha$ is dimensionless.

\subsection{Establishing the State Function to Determine Turning Radius}

The turning radius of the vehicle $R$ is a function depending on the velocity $\mathrm{v}$ and steering angle $\delta$.

$$
R=f(v, \delta)
$$

From the graph in Figure 2, the turning radius of the vehicle $R$ can be calculated as follows:

$$
\left\{\begin{array}{l}
v_{i}=v_{0}+\mu_{1} i \\
R_{i}=R_{0}+\alpha \frac{i(i+1)}{2}
\end{array}\right.
$$

First, the factor $i$ needs to be determined based on velocity $v_{i}$ at $i$ time. Then, to calculate $R_{i}$ by using the formula (10), it is necessary to determine the coefficients $R_{0}$ and $\alpha$.

The coefficients $R_{0}$ depends on the steering angle $\delta$ at $j$ time. This value can be determined as follows:

$$
\left\{\begin{array}{l}
\delta_{j}=\delta_{M}-\mu_{2} j \\
R_{O j}=R_{O M}+\beta \frac{j(j+1)}{2}
\end{array}\right.
$$

Finally, the coefficient $\alpha$ needs to be determined. The coefficient $\alpha$ depends on the steering angle $\delta$ at $k$ time, this value can be determined through the formula below:

$$
\left\{\begin{array}{l}
\delta_{k}=\delta_{M}-\mu_{3} k \\
\alpha_{k}=\alpha_{M}+\gamma \frac{k(k+1)}{2}
\end{array}\right.
$$

The remaining coefficients can be used in Table 2 .

Table 2. The determined coefficients

\begin{tabular}{|c|c|c|}
\hline Symbol & Value & Unit \\
\hline$\mu_{1}$ & 5 & \\
\hline$\mu_{2}$ & 0.5 & \\
\hline$\mu_{3}$ & 1 & \\
\hline$\beta$ & 2.2 & \\
\hline$\gamma$ & 0.15 & $\mathrm{~km} / \mathrm{h}$ \\
\hline$\alpha_{M}$ & 0.55 & $\mathrm{degree}$ \\
\hline$v_{0}$ & 5 & $\mathrm{~m}$ \\
\hline$\delta_{M}$ & 5 & \\
\hline$R_{O M}$ & 33 & \\
\hline
\end{tabular}

\subsection{The Tolerance of the Result Calculation}

The results of the calculation process by using state function (case 1) and the simulation method (case 2) are given as in Table 3, Table 4, and Table 5. In general, tolerance is quite small (less than $3 \%$ ).

Table 3. Turning radius.

\begin{tabular}{|c|c|c|c|c|c|c|c|c|c|}
\hline & \multicolumn{3}{|c|}{ Steering angle $\delta=\mathbf{1 . 0}^{\mathbf{0}}$} & \multicolumn{3}{c|}{ Steering angle $\boldsymbol{\delta}=\mathbf{1 . 5}^{\mathbf{0}}$} & \multicolumn{3}{c|}{ Steering angle $\boldsymbol{\delta}=\mathbf{2 . \mathbf { 0 } ^ { \mathbf { 0 } }}$} \\
\hline $\begin{array}{c}\text { Velocity } \\
(\mathbf{k m} / \mathbf{h})\end{array}$ & Case 1 & Case 2 & Tolerance (\%) & Case 1 & Case 2 & Tolerance (\%) & Case 1 & Case 2 & Tolerance (\%) \\
\hline 5 & 112.20 & 111.18 & 0.91 & 94.60 & 93.72 & 0.93 & 79.20 & 77.68 & 1.92 \\
\hline 10 & 114.25 & 113.55 & 0.61 & 96.33 & 95.46 & 0.90 & 80.65 & 79.82 & 1.03 \\
\hline 15 & 118.35 & 117.25 & 0.93 & 99.79 & 97.74 & 2.06 & 83.55 & 81.44 & 2.53 \\
\hline 20 & 124.50 & 122.13 & 1.90 & 104.99 & 102.15 & 2.70 & 87.90 & 85.79 & 2.40 \\
\hline 25 & 132.70 & 130.45 & 1.70 & 111.91 & 109.88 & 1.82 & 93.70 & 91.23 & 2.64 \\
\hline 30 & 142.95 & 139.35 & 2.52 & 120.57 & 117.89 & 2.22 & 100.95 & 98.1 & 2.82 \\
\hline 35 & 155.25 & 153.22 & 1.31 & 130.96 & 128.43 & 1.93 & 109.65 & 107.85 & 1.64 \\
\hline 40 & 169.60 & 166.98 & 1.54 & 143.08 & 141.02 & 1.44 & 119.80 & 116.88 & 2.44 \\
\hline 45 & 186.00 & 184.85 & 0.62 & 156.93 & 153.22 & 2.36 & 131.40 & 129.01 & 1.82 \\
\hline 50 & 204.45 & 201.36 & 1.51 & 172.51 & 170.12 & 1.38 & 144.45 & 142.25 & 1.52 \\
\hline 55 & 224.95 & 221.88 & 1.36 & 189.82 & 187.43 & 1.26 & 158.95 & 157.56 & 0.87 \\
\hline 60 & 247.50 & 246.54 & 0.39 & 208.86 & 206.55 & 1.11 & 174.90 & 172.91 & 1.14 \\
\hline 65 & 272.10 & 270.03 & 0.76 & 229.64 & 226.48 & 1.37 & 192.30 & 189.92 & 1.24 \\
\hline 70 & 298.75 & 293.55 & 1.74 & 252.14 & 250.41 & 0.69 & 211.15 & 207.98 & 1.50 \\
\hline 75 & 327.45 & 325.32 & 0.65 & 276.38 & 273.84 & 0.92 & 231.45 & 228.15 & 1.43 \\
\hline 80 & 358.20 & 354.48 & 1.04 & 302.35 & 298.98 & 1.11 & 253.20 & 249.73 & 1.37 \\
\hline
\end{tabular}


Table 4. Turning radius.

\begin{tabular}{|c|c|c|c|c|c|c|c|c|c|}
\hline \multirow[b]{2}{*}{$\begin{array}{c}\text { Velocity } \\
(\mathbf{k m} / \mathbf{h})\end{array}$} & \multicolumn{3}{|c|}{ Steering angle $\delta=2.5^{\circ}$} & \multicolumn{3}{|c|}{ Steering angle $\delta=3.0^{\circ}$} & \multicolumn{3}{|c|}{ Steering angle $\delta=3.5^{\circ}$} \\
\hline & Case 1 & Case 2 & Tolerance $(\%)$ & Case 1 & Case 2 & Tolerance (\%) & Case 1 & Case 2 & Tolerance $(\%)$ \\
\hline 5 & 66.00 & 64.98 & 1.55 & 55.00 & 53.95 & 1.91 & 46.20 & 45.18 & 2.21 \\
\hline 10 & 67.21 & 65.73 & 2.20 & 56.00 & 54.88 & 2.00 & 47.03 & 46.03 & 2.13 \\
\hline 15 & 69.62 & 68.43 & 1.71 & 58.00 & 57.02 & 1.69 & 48.69 & 47.55 & 2.35 \\
\hline 20 & 73.24 & 71.87 & 1.87 & 61.00 & 59.43 & 2.57 & 51.19 & 49.98 & 2.36 \\
\hline 25 & 78.06 & 75.98 & 2.67 & 65.00 & 63.25 & 2.69 & 54.51 & 53.13 & 2.54 \\
\hline 30 & 84.09 & 83.17 & 1.10 & 70.00 & 69.1 & 1.29 & 58.67 & 57.76 & 1.55 \\
\hline 35 & 91.33 & 90.43 & 0.99 & 76.00 & 74.48 & 2.00 & 63.66 & 61.98 & 2.63 \\
\hline 40 & 99.78 & 97.75 & 2.03 & 83.00 & 81.55 & 1.75 & 69.48 & 67.58 & 2.73 \\
\hline 45 & 109.43 & 107.82 & 1.47 & 91.00 & 89.21 & 1.97 & 76.13 & 75.89 & 0.31 \\
\hline 50 & 120.28 & 117.88 & 2.00 & 100.00 & 97.28 & 2.72 & 83.61 & 81.26 & 2.81 \\
\hline 55 & 132.34 & 131.12 & 0.92 & 110.00 & 107.73 & 2.06 & 91.92 & 89.55 & 2.58 \\
\hline 60 & 145.61 & 143.26 & 1.62 & 121.00 & 118.45 & 2.11 & 101.06 & 99.83 & 1.22 \\
\hline 65 & 160.09 & 158.73 & 0.85 & 133.00 & 130.89 & 1.59 & 111.04 & 109.18 & 1.67 \\
\hline 70 & 175.77 & 173.27 & 1.42 & 146.00 & 142.56 & 2.36 & 121.84 & 119.84 & 1.64 \\
\hline 75 & 192.66 & 189.92 & 1.42 & 160.00 & 157.72 & 1.43 & 133.48 & 129.87 & 2.71 \\
\hline 80 & 210.75 & 208.85 & 0.90 & 175.00 & 172.29 & 1.55 & 145.95 & 143.12 & 1.94 \\
\hline
\end{tabular}

Table 5. Turning radius.

\begin{tabular}{|c|c|c|c|c|c|c|c|c|c|}
\hline \multirow[b]{2}{*}{$\begin{array}{l}\text { Velocity } \\
(\mathbf{k m} / \mathbf{h})\end{array}$} & \multicolumn{3}{|c|}{ Steering angle $\delta=4.0^{\circ}$} & \multicolumn{3}{|c|}{ Steering angle $\delta=4.5^{\circ}$} & \multicolumn{3}{|c|}{ Steering angle $\delta=5.0^{\circ}$} \\
\hline & Case 1 & Case 2 & Tolerance $(\%)$ & Case 1 & Case 2 & Tolerance (\%) & Case 1 & Case 2 & Tolerance (\%) \\
\hline 5 & 39.60 & 38.76 & 2.12 & 35.20 & 34.46 & 2.10 & 33.00 & 32.16 & 2.55 \\
\hline 10 & 40.30 & 39.45 & 2.11 & 35.81 & 35.02 & 2.20 & 33.55 & 33.12 & 1.28 \\
\hline 15 & 41.70 & 40.72 & 2.35 & 37.02 & 36.87 & 0.40 & 34.65 & 34.01 & 1.85 \\
\hline 20 & 43.80 & 42.98 & 1.87 & 38.84 & 37.96 & 2.26 & 36.30 & 35.27 & 2.84 \\
\hline 25 & 46.60 & 45.72 & 1.89 & 41.26 & 40.45 & 1.97 & 38.50 & 37.47 & 2.68 \\
\hline 30 & 50.10 & 48.79 & 2.61 & 44.29 & 43.08 & 2.74 & 41.25 & 40.16 & 2.64 \\
\hline 35 & 54.30 & 52.88 & 2.62 & 47.93 & 46.97 & 2.01 & 44.55 & 43.65 & 2.02 \\
\hline 40 & 59.20 & 58.73 & 0.79 & 52.18 & 50.87 & 2.50 & 48.40 & 47.86 & 1.12 \\
\hline 45 & 64.80 & 63.12 & 2.59 & 57.03 & 56.13 & 1.57 & 52.80 & 51.36 & 2.73 \\
\hline 50 & 71.10 & 70.15 & 1.34 & 62.48 & 61.04 & 2.31 & 57.75 & 56.03 & 2.98 \\
\hline 55 & 78.10 & 77.95 & 0.19 & 68.54 & 66.78 & 2.57 & 63.25 & 62.19 & 1.68 \\
\hline 60 & 85.80 & 83.34 & 2.87 & 75.21 & 73.15 & 2.74 & 69.30 & 67.55 & 2.53 \\
\hline 65 & 94.20 & 93.26 & 1.00 & 82.49 & 80.19 & 2.79 & 75.90 & 73.74 & 2.85 \\
\hline 70 & 103.30 & 101.15 & 2.08 & 90.37 & 89.72 & 0.72 & 83.05 & 81.67 & 1.66 \\
\hline 75 & 113.10 & 109.99 & 2.75 & 98.86 & 96.13 & 2.76 & 90.75 & 88.53 & 2.45 \\
\hline 80 & 123.60 & 121.13 & 2.00 & 107.95 & 105.34 & 2.42 & 99.00 & 97.85 & 1.16 \\
\hline
\end{tabular}




\section{Conclusions}

The turning radius of the vehicle is a value depending on the velocity and steering angle. As the velocity increases, the turning radius will increase. When the steering angle increases, the turning radius will decrease. The change of the turning radius according to the above two parameters is nonlinear. When the velocity increases, the turning radius will increase. When the steering angle increases, the turning radius will decrease.

This research established the state function to determine the turning radius at different velocities and steering angles. With input data are velocity and steering angle, necessary to use the three of the state functions (10), (11), and (12) to determine the value of the turning radius.

The value of the turning radius is calculated by the state function equivalent to the result of the simulation process, the tolerance is very small (less than 3\%). This method helps the calculation process take place faster under different conditions without using dynamic models to simulate. Therefore, the algorithms can be developed to predict the vehicle's trajectory in a very short time to improve the safety and stability of the vehicle. The results of this research are the basis for the development of other computational researches in the future of autonomous vehicles. Besides, the dangerous trajectory of the vehicle when steering can be predicted in advance based on the input parameters.

\section{REFERENCES}

[1] J. Chi, S. Liang, L. Cornelia, and E. Arno, Vehicle Side Slip Angle Observation with Road Friction Adaptation, International Federation of Automatic Control, 3406-3411, 2017. DOI: 10.1016/j.ifacol.2017.08.593

[2] N. T. Hoan, Design and Calculate of the Vehicle, Vietnam Education Publishing, Vietnam, 2020.

[3] B. Dainis, Research in Kinematics of Turn for Vehicles and Semitrailers, International Scientific Conference, 177-182, 2008.

[4] A. A. Markina, S. N. Chepkasov, and Berezhnaya, Kinematic Study of the Four-wheeled Steering System, The Russian Automobile and Highway Industry Journal, Vol.16, No.5, 581-591, $2019 . \quad$ DOI: 10.26518/2071-7296-2019-5-580-591.

[5] V. Arvind, Optimizing the Turning Radius of a Vehicle Using Symmetric Four-wheel Steering System, International Journal of Scientific \& Engineering Research, Vol.4, No.12, 2177-2184, 2013.

[6] K. G. Bhavesh, G. J. Nilesh, B. P. Hardik, and B. K. Pritesh, Optimization of Steering System for Four-wheel Vehicle, International Journal of Mechanical and Production Engineering, Vol.6, No.8. 52-62, 2018.

[7] J. Haobin, T. Huan, H. Yiding, and T. Bin, Research on
Control of Intelligent Vehicle Human-Simulated Steering System Based on HSIC, Applied Sciences, Vol.9, No.5, 1-15, 2019. DOI: 10.3390/app9050905

[8] A. Singh, A. Kumar, R. Chaudhary, and R. C. Singh, Study of 4 Wheels Steering Systems to Reduce Turning Radius and Increase Stability, International Conference of Advance Research and Innovation, 96-102, 2014.

[9] K. Zhang, J. Wang, N. Chen, M. Cao, and G. Yin, Design of a Cooperative V2V Trajectory-planning Algorithm for Vehicles Driven on a Winding Road with Consideration of Human Drivers' Characteristics, IEEE Access, Vol.7, 131135-131147, 2019. DOI: 10.1109/ACCESS.2019.2941 053

[10] M. Andras, N. Balazs, and G. Peter, Integrated Vehicle Control of in-wheel Electric Vehicle, Periodica Polytechnica Transportation Engineering, Vol.42, No.1, 19-25, 2014. DOI: 10.3311/PPtr.7280

[11] B. Karl, and M. Fredrik, Hierarchical Predictive Control for Ground-vehicle Maneuvering, 2015 American Control Conference, 2771-2776, 2015. DOI: 10.1109/ACC.2015.7 171154

[12] U. Erdem, and E. Veysel, Development of a Trajectory Following Vehicle Control Model, Advances in Mechanical Engineering. Vol.8, No.5, 1-11, 2016. DOI: 10.1177/1687 814016650832

[13] N. T. Anh, and H. T. Binh, Research on Determining the Limited Roll Angle of Vehicle, Lecture Notes in Networks and Systems, Vol. 104, 613-619, 2020. DOI: 10.1007/978-3-030-37497-6_70

[14] N. T. Anh, and H. T. Binh, Research on Dynamic Vehicle Model Equipped Active Stabilizer Bar, Advances in Science, Technology and Engineering System Journal, Vol.4, No.4, 271-275, 2019. DOI: 10.25046/aj040434

[15] H. B. Pacejka, Tire and Vehicle Dynamics 3rd, Butterworth-Heinemann Publishing, Oxford, 2012. 